\title{
Advances in nutritional status of patients with connective tissue- associated pulmonary hypertension
}

\author{
Jiang Jinyan ${ }^{1}$, Li Jin ling ${ }^{1}$, Chengjie ${ }^{1}$ \\ ${ }^{1}$ Shanghai Lung Hospital Shanghai 200433
}

\begin{abstract}
Connective Tissue Disease (CTD) is an autoimmune disease involving connective tissues, including sjogren syndrome (pSS), rheumatoid arthritis (RA), systemic sclerosis (SSc), mixed connective tissue disease (MCTD), systemic lupus erythematosus (SLE), etc. [1,2]. Pulmonary Arterial Hypertension $(\mathrm{PAH})$ refers to a pathological condition in which the pulmonary artery pressure rises above a certain threshold, causing right heart failure and death [3]. PAH refers to the mean Pulmonary Artery Pressure (mPAP) of greater than $25 \mathrm{mmHg}$ and the pulmonary wedge pressure (PAWP) of less than $15 \mathrm{mmhg}$ under right heart catheterization (RHC) at rest [4,5]. The only criterion for examining pulmonary hypertension is right cardiac catheterization. Connective tissue disease-associated pulmonary hypertension (CTD-PAH) is a rare complication of $\mathrm{PAH}$, characterized by high mortality, venous and cardiac involvement and poor prognosis [6-8].
\end{abstract}

\section{Epidemiology of connective tissue- associated pulmonary hypertension}

Nutrition and health conditions have certain impact on the occurrence of diseases. Studies have shown that malnutrition is an important risk factor for the death of CTD-PAH patients [9]. Nutritional risk refers to the existing or potential risks related to nutritional factors that cause adverse clinical outcomes of patients. Nutritional risk screening could provide references for nutritional support and predict the prognosis of clinical outcomes [10, 11]. Malnutrition could cause changes of body fat mass and somatic cell mass as a result of malnutrition intake or absorption, ultimately leading to compromised clinical outcomes. Domestic and foreign data show that malnutrition or malnutrition is common in patients treated in hospital, with an incidence rate of up to $40-60 \%$ [12]. Screening the nutritional risks of admitted patients and providing early nutritional intervention for patients with such risks could effectively improve the health conditions of patients and shorten the time they spend in hospital.

The subtypes of CTD-PAH, including SLE-PAH, pSS$\mathrm{PAH}, \mathrm{RA}-\mathrm{PAH}, \mathrm{MCTD}-\mathrm{PAH}$ and SSc-PAH are commonly found in China, accounting for $48 \%, 16 \%, 3 \%$, $9 \%$ and $6 \%$ respectively (Figure 1)[13]. The registered study by Chinese SLE Treatment And Research Group [14] found that the incidence of PAH-SLE was $3.9 \%$, lower than that of joint involvement in SLE (54.5\%), lupus nephritis (47.4\%), blood system involvement in SLE (56.1\%), as well as the neuropsychiatric lupus (4.8\%), as shown in Figure 2A. The results of a large-scale study showed that the total mortality rate of SLE diagnosed with $\mathrm{PAH}$ is up to $25-50 \%$ in 2 years [15]. Pulmonary hypertension is a major cause of death in patients with systemic lupus erythematosus, and a risk factor for poor prognosis of patients with SLE. Studies by Xu Dong (2011) and others [16] showed that the incidence of SSc-PAH was $20.2 \%$, far lower than that of pulmonary interstitial fibrosis $(58.8 \%)$, gastroesophageal reflux $(56.3 \%)$ and fingertip ulcer $(31.3 \%$ ) caused by SSc. In addition, pSS, RA and MCTD can involve pulmonary vessels and cause pulmonary hypertension. Therefore, $\mathrm{PAH}$, as a complication of CTD, has a lower incidence and a higher mortality compared with other complications. However, as the occult onset of PAH presents no special clinical manifestations in early the stage, it is very likely to be misdiagnosed, forming an important factor affecting the prognosis of CTD patients. 


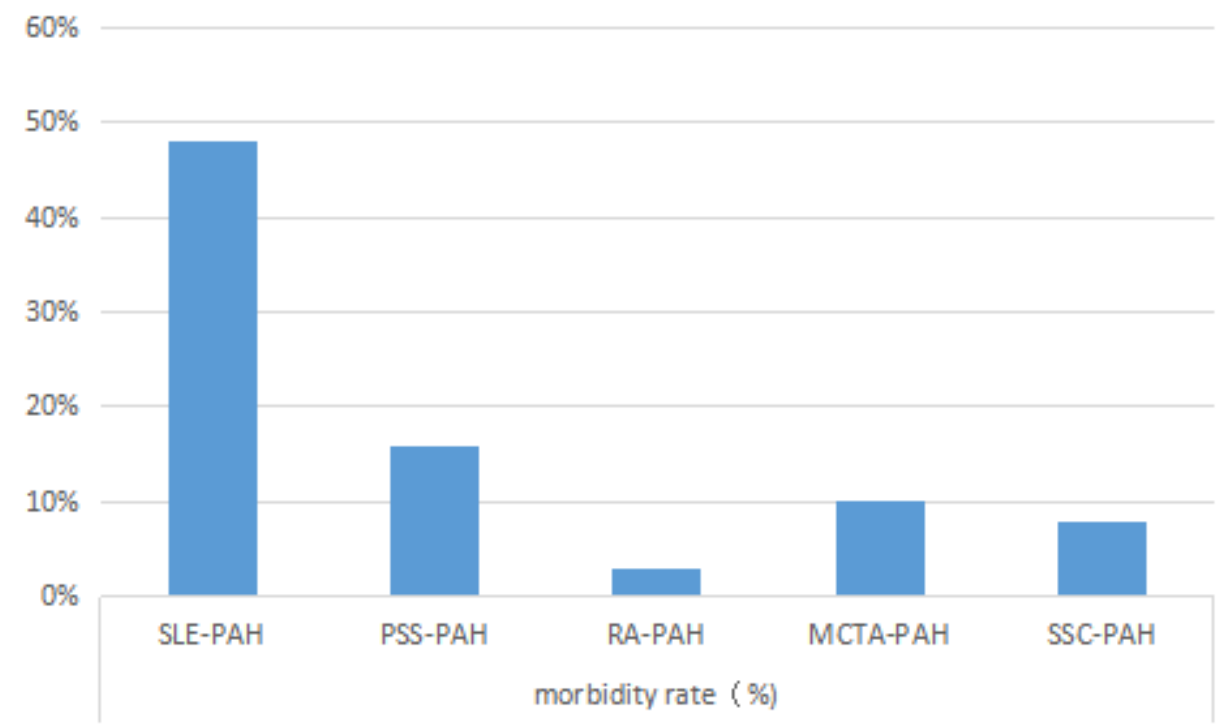

Figure 1 Prevalence of PAH in CTD-PAH in China
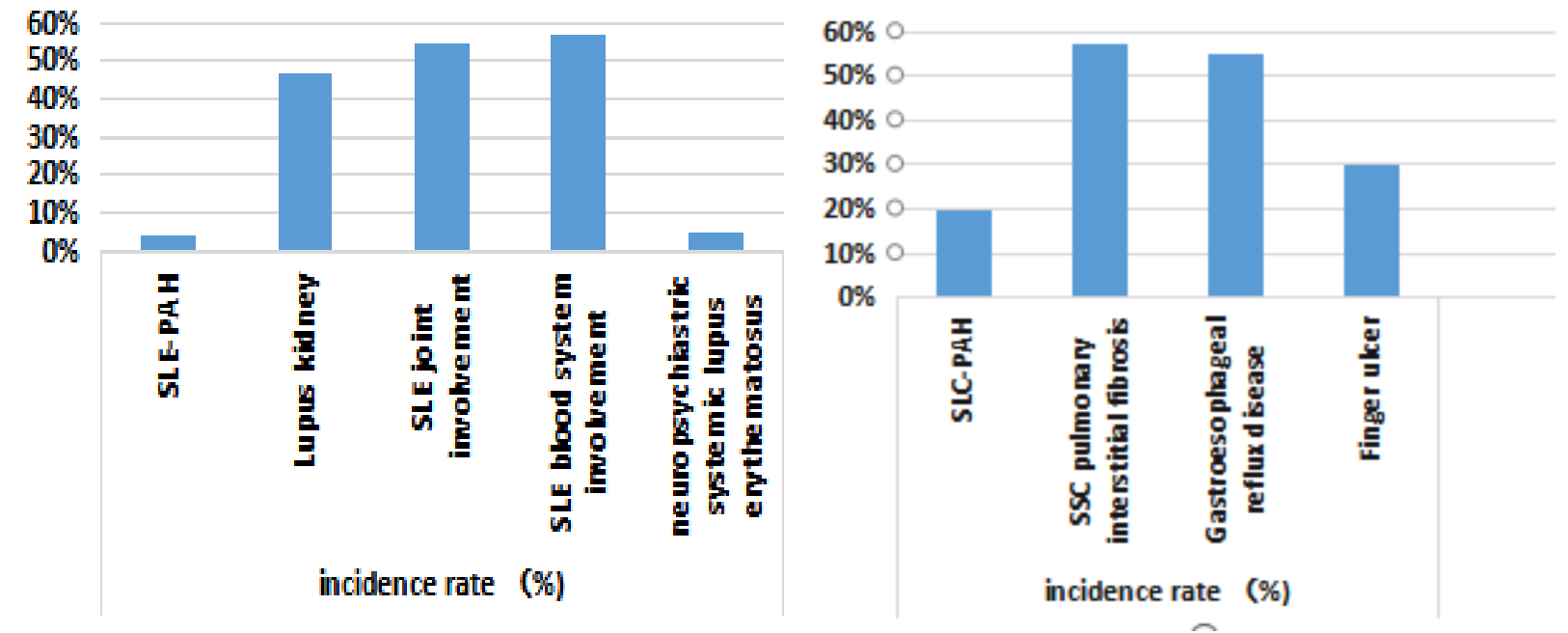

Figure 2 Incidence of SLE-PAH, SSc-PAH and Related Diseases (Diagram A presents the incidence of SLE-PAH and related diseases; (Diagram B presents the incidence of SSc-PAH and related diseases)

\section{Mechanism of malnutrition in patients with connective tissue-related pulmonary hypertension}

CTD patients with immune function disorders could be further weakened by the use of glucocorticoid and immunosuppressant as part of the treatment, with a great likelihood of concurrent infections, a leading cause of death for CTD patients [17]. During the treatment, longterm use of glucocorticoids will cause osteoporosis in patients. Therefore, patients need to intake more nutrition and improve immunity during treatment. Related studies have shown that malnutrition is a common complication in CTD-PAH patients mainly as a result of decreased nutrition intake of CTD-PAH patients who have a greater demand for energy with the progress of the disease. It is likely to take place in the presence of the imbalance [18]. CTD involves pulmonary vessels, causing pulmonary vasoconstriction and increasing pulmonary circulation resistance and pulmonary artery pressure. With the progress of disease, rising respiratory muscle load would cause respiratory muscle fatigue and compromise ventilation functions, increasing oxygen consumption and nutritional needs of CTD-PAH patients. Besides, factors such as hypoxemia, right ventricular dysfunction and long-term medication in CTD-PAH patients would cause further loss of appetite and aggravate the risk of malnutrition [19].

\section{Study on the nutritional conditions of patients with connective tissue-related arterial hypertension}

There are few studies on nutritional conditions of patients with connective tissue-related arterial hypertension abroad. A literature about malnutrition in patients with pulmonary hypertension suggests that although malnutrition is often found in PAH, little is known about the effectiveness of nutrition and lifestyle intervention in PAH patients [20]. A 
study on the correlation between Vitamin C deficiency and pulmonary hypertension proved that Vitamin $\mathrm{C}$ deficiency is the mechanism leading to pulmonary hypertension, which can be effectively alleviated by the intake of Vitamin C. Therefore, it is of great significance to determine the possibility of Vitamin $\mathrm{C}$ deficiency in PAH patients in a proper clinical environment [21]. Also, there is a literature about PAH that focused only on the diagnosis and treatment of PAH without nutritional intervention [22]. Vinke et al. [23] pointed out that although the data about nutritional conditions and micronutrient deficiency of PAH is not widely available yet, some studies have proved that nutritional intervention aiming at improving nutritional deficiency and metabolic changes may help alleviate symptoms of the disease, and called for systematic analysis of the nutritional conditions of PAH patients as part of studies on nutritional interventions.

At present, there are few studies on the nutritional conditions of CTD-PAH patients in China, with no literatures on nutritional risk screening and nutritional intervention of CTD-PAH patients. In developed countries in Europe and America, nutrition diagnosis and treatment has been widely applied in clinical practices. For example, $45-55 \%$ of inpatients receiving surgeries in America have nutrition diagnosis and treatment, with the nutrition diagnosis rate of children reaching 43-80\% [24]. However, nutrition diagnosis started late in China, with much room of improvement in the nutrition diagnosis and intervention system. Malnutrition is an important starting point for nutrition diagnosis and treatment. Inadequate supply of nutrients, conditions of disease and inflammatory reaction can lead to increased catabolism and disorder of metabolism. Only by effectively controlling the primary disease can we help patients recover when the inflammatory reaction disappears and the body goes to the anabolic stage.

\section{Discussion and Prospect}

Multi-disciplinary team (MDT) is a clinical treatment model utilizing and integrating medical resources, thus giving full play to the advantages of multi-disciplinary medical staff, who provide diagnosis and treatment to patients at the center of the model. This model has proved highly efficient in delivering quality services to patients [25]. The MDT is a newly emerging model in China, a goal for medical institutions to explore and develop. Studies showed that the MDT can improve the selfmanagement behaviors of patients, reduce the occurrence of postoperative complications and improve the prognosis of patients [26,27]. At present, international and domestic medical institutions are working hard to explore the possibility of MTD, which not only integrates medical resources but also provides patients with individualized and better diagnosis and treatment. Also, it promotes the common development of related specialties in hospitals. In 2015, the Guidelines for Diagnosis and Treatment of Pulmonary Hypertension by European Society of Cardiology defined CTD-PAH as an interdisciplinary disease. In order to identify CTD-PAH in the early stage and offer prompt treatment, close cooperation among cardiology, respiratory and rheumatology departments is needed [28].

In conclusion, CTD-PAH patients are characterized by high mortality and poor prognosis. Malnutrition is a common complication of CTD-PAH patients. The risk of malnutrition would increase the incidence of complications and mortality, thus pressuring patients. At present, there are few studies on the nutritional conditions of connective tissue-related pulmonary hypertension in China, without standardized procedures of nutritional diagnosis and intervention. Therefore, it is necessary to further analyze the nutritional conditions of CTD-PAH patients, and adopt multidisciplinary and precise nutrition interventions based on the results of analysis so as to lower nutritional risks, improve malnutrition, reduce disease burden and improve clinical outcomes. On the basis of studies on the nutritional conditions of CTD-PAH patients, this study tested and verified the efficiency of nutritional intervention of CTD-PAH patients through the cooperation of clinicians, nutritionists and nursing staff, providing references for nutritional intervention of CTDPAH patients. Also, it further enriched the MDT model and highlighted the significance of nutrition in disease diagnosis and treatment.

\section{References}

1. Galiè N, Humbert M, Vachiery JL, et al. ESC Scientific Document Group. 2015 ESC/ERS Guidelines for the diagnosis and treatment of pulmonary hypertension: The Joint Task Force for the Diagnosis and Treatment of Pulmonary Hypertension of the European Society of Cardiology (ESC) and the European Respiratory Society (ERS): Endorsed by: Association for European Paediatric and Congenital Cardiology (AEPC), International Society for Heart and Lung Transplantation (ISHLT). Eur Heart J. 2016 Jan 1;37(1):67-119.

2. Shiboski CH, Shiboski SC, Seror R, et al. International Sjögren's Syndrome Criteria Working Group. 2016 American College of Rheumatology/European League Against Rheumatism Classification Criteria for Primary Sjögren's Syndrome: A Consensus and Data-Driven Methodology Involving Three International Patient Cohorts. Arthritis Rheumatol. 2017 Jan;69(1):35-45.

3. Wang Han. Pulmonary Hypertension Associated with Connective Tissue Diseases [J]. Advances in Cardiovascular Diseases, 2016, 37 (06): 643-646.

4. Hill NS, Cawley MJ, Heggen-Peay CL. New Therapeutic Paradigms and Guidelines in the Management of Pulmonary Arterial Hypertension. J Manag Care Spec Pharm. 2016 Mar;22(3 Suppl A):S3-21.

5. Huang WC, Hsu CH, Sung SH, et al; TSOC pulmonary hypertension committee. 2018 TSOC guideline focused update on diagnosis and treatment of pulmonary arterial hypertension. J Formos Med Assoc. 2019 Dec;118(12):1584-1609. 
6. Wang Hui, Zhang Na, Zhao Yin, et al. Clinical Characteristics of Arterial Pulmonary Hypertension Associated with Connective Tissue Disease [J]. Journal of Tianjin Medical University, 2020, 26(01): 32-34.

7. Zhao Jiuliang, Wang Qian, Li Mengtao, et al. Progress in Treatment of Pulmonary Hypertension Associated with Connective Tissue Disease [J]. Clinical Medication Journal, 2018,16 (02): 9-12.

8. Chang Zhifang, Feng Chenglong, Wang Yongfu. Progress in Diagnosis and Treatment of Pulmonary Hypertension Associated with Connective Tissue Disease [J]. Chinese General Medicine, 2017, 20 (S2): 194-196.

9. Dong Fang, Zhang Yihui, Guo Jian, et al. Study on the Correlation between Nutritional Risks and Exercise Tolerance in Patients with Idiopathic Pulmonary Hypertension [J]. Diagnostic Theory and Practice, 2018,17 (06): 664-669.

10. $\mathrm{Mu}$ Yan, Xu Chenyu. Influence of Nutritional Risks and Nutritional Support on Clinical Outcome of Inpatients [J]. Journal of Nursing Science, 2016, 31 (05): 87-90.

11. Li Suyun, Yu Jiaohua, Zeng Li, et al. Nutritional Risk Screening and Nutritional Support Analysis of Inpatients [J]. Journal of Nursing Science, 2016,31 (21): 99-102.

12. Ulibarri JI, Burgos R, Lobo G, et al. Recommendations for assessing the hyponutrition risk in hospitalized patients Nutr Hosp.2009, 24(4):467-472.

13. M Li, Q Wang, J Zhao, et al. Chinese SLE Treatment and Research group (CSTAR) registry: II. Prevalence and risk factors of pulmonary arterial hypertension in Chinese patients with systemic lupus erythematosus[J]. Lupus, 2014, 23(10).

14. Li M, Zhang W, Leng $X$, et al. Chinese SLE Treatment and Research group(CSTAR) registry:I. Major clinical characteristics of Chinese patients with systemic lupus erythematosus[J]. Lupus, 2013, 22(11):1192-1199.

15. Zhang Zhuoli. Pulmonary Arterial Hypertension Associated with Systemic Lupus Erythematosus [J]. Chinese Journal of Practical Internal Medicine, 2015,35 (10): 807-809, 813

16. Xu Dong, Li Mengtao, Hou Yong, et al. Analysis of Clinical Manifestations of Patients with Systemic Sclerosis- Data from Chinese EUSTAR Database [J]. Chinese Journal of Rheumatology, 2011, 15(7): 455459.

17. Zhang Xiyue, Su Zhenzhong, Dong Chunling, $\mathrm{Xu}$ Yingyu, et al. A Case Study of Acute Progression of Pulmonary Hypertension Associated with Connective Tissue Disease Induced by Infection [J]. Chinese Journal of Laboratory Diagnosis, 2018, 22 (07): 12981299.

18. Dong Fang, Zhang Yihui, Guo Jian, et al. Study on the Correlation between Nutritional Risks and Exercise
Tolerance in Patients with Idiopathic Pulmonary Hypertension [J]. Diagnostic Theory and Practice, 2018,17 (06): 664-669.

19. Mu Yan, Xu Chenyu. Influence of Nutritional Risks and Nutritional Support on Clinical Outcomes of Inpatients [J]. Journal of Nursing Science, 2016, 31(5): 87-90.

20. Kwant CT, Ruiter G, Noordegraaf AV. Malnutrition in pulmonary arterial hypertension: a possible role for dietary intervention. Curr Opin Pulm Med. 2019,25(5):405-409.

21. Gayen SK, Abdelrahman AA, Preston IR, Petit RD, Hill NS. Vitamin C Deficiency-Induced Pulmonary Arterial Hypertension. Chest. 2020,157(2):21-23.

22. Barnett CF, Alvarez P, Park MH. Pulmonary Arterial Hypertension: Diagnosis and Treatment[J]. Cardiology Clinics, 2016, 34(3):375-389.

23. Vinke P, Jansen SM, Witkamp R F, et al. Increasing quality of life in pulmonary arterial hypertension:is there a role for nutrition[J]. Heart failure reviews,2018,23(5).

24. Ren Jianan. Challenges and Opportunities of Clinical Nutrition Therapy [J]. Parenteral and Enteral Nutrition, 2016, 23 (1): 13.

25. Di Jianzhong, Li Kun, Ren Qinggui, et al. Research Progress of Multi-disciplinary Team Model in Clinical Application [J]. Chinese Hospital, 2016,20 (01): 79-80.

26. Cheng Di, Guo Xiao, Dai Yi. Application of Multidisciplinary Team Model in Patients Undergoing Radical Mastectomy [J]. Qilu Journal of Nursing, 2019, 25 (24): 41-43.

27. Tao Jingnan. Application of Multi-disciplinary Team Model in Patients with Rectal Cancer Undergoing Mile's Surgery [J]. General Nursing, 2020,18 (13): 1611-1613.

28. Chang Zhifang, Feng Chenglong, Wang Yongfu. Progress in Diagnosis and Treatment of Pulmonary Hypertension Associated with Connective Tissue Disease [J]. Chinese General Medicine, 2017,20 (S2): 194-196. 